\title{
An ANN Based Hybrid Chemistry Framework for Complex Fuels
}

\author{
Rishikesh Ranade ${ }^{\mathrm{a}}$, Sultan Alqahtani ${ }^{\mathrm{a}}$, Aamir Farooq ${ }^{\mathrm{b}}$, Tarek Echekki ${ }^{\mathrm{a}, 1}$ \\ ${ }^{\mathrm{a}}$ Department of Mechanical and Aerospace Engineering, North Carolina State University, Raleigh, \\ NC 27695-7910, USA \\ ${ }^{\mathrm{b}}$ King Abdullah University of Science and Technology, Clean Combustion Research Center, Physical \\ Sciences and Engineering Division, Thuwal 23955, Saudi Arabia
}

\begin{abstract}
The oxidation chemistry of complex hydrocarbons involves large mechanisms with hundreds or thousands of chemical species and reactions. For practical applications and computational ease, it is desirable to reduce their chemistry. To this end, high-temperature fuel oxidation for large carbon number fuels may be described as comprising two steps, fuel pyrolysis and small species oxidation. Such an approach has recently been adopted as 'hybrid chemistry' or HyChem to handle hightemperature chemistry of jet fuels by utilizing time-series measurements of pyrolysis products. In the approach proposed here, a shallow Artificial Neural Network (ANN) is used to fit temporal profiles of fuel fragments to directly extract chemical reaction rate information. This information is then correlated with the species concentrations to build an ANN-based model for the fragments' chemistry during the pyrolysis stage. Finally, this model is combined with a $\mathrm{C}_{0}-\mathrm{C}_{4}$ chemical mechanism to model high-temperature fuel oxidation. This new hybrid chemistry approach is demonstrated using homogeneous chemistry calculations of $n$-dodecane $\left(n-\mathrm{C}_{12} \mathrm{H}_{26}\right)$ oxidation. The experimental uncertainty is simulated by introducing realistic noise in the data. The comparison shows a good agreement between the proposed ANN hybrid chemistry approach and detailed chemistry results.
\end{abstract}

Keywords: Chemistry reduction; artificial neural networks; hydrocarbon oxidation; pyrolysis.

\footnotetext{
${ }^{1}$ Corresponding Author. Address: Department of Mechanical and Aerospace Engineering, North Carolina State University, 911 Oval Drive, Campus Box 7910, Engineering Building III, Room 3252, Raleigh, NC 27695-7910, USA. Fax: +1 919515 7968, E-mail address: techekk@,ncsu.edu (T. Echekki).
} 


\section{Introduction}

The chemical kinetics of larger carbon number fuels involves hundreds to thousands of species and tens of thousands of elementary chemical reactions. Handling the sheer complexity of these mechanisms is not practical beyond 0-D unsteady or 1-D steady simulations. Strategies to reduce this complexity involves the implementation of an array of chemistry reduction tools to achieve a tractable mechanism in the form of skeletal or reduced chemical descriptions [1]. High-temperature oxidation for large hydrocarbons undergoes two reasonably decoupled steps: 1) a short fuel pyrolysis step (of the order of $100 \mu \mathrm{s}$ ) resulting in the formation of smaller fragments, followed by 2) a longer oxidation step for these fragments [2]. This decoupling enables several approaches to describe the pyrolysis step in a much simpler formulation [2 -4].

More recently, Wang and co-workers [5-11] proposed a hybrid chemistry (HyChem) reduction model based on this decoupling. For fuel pyrolysis, Wang et al. [5-11] proposed a lumped model represented by a set of global steps for $\mathrm{C}-\mathrm{C}$ fission and radical abstraction reactions. The second step representing oxidation is modeled using a foundational $\mathrm{C}_{0}-\mathrm{C}_{4}$ chemistry mechanism to describe the oxidation of fuel fragments. A key innovation in the HyChem approach is the reliance on experimental measurements of fragments' time-histories and yields to construct a reduced chemical representation even for fuels for which detailed chemical mechanisms are not available. With experimental measurements of the key fuel fragments, the stoichiometric coefficients and rate constants for the global reactions are determined through an optimization approach.

While the HyChem approach [5-11] represents an efficient strategy for chemistry reduction of complex fuels, other approaches built on the same premises may be adopted with potential advantages and drawbacks. One of the motivations towards exploring additional strategies is whether useful information can be extracted directly from temporal measurements for key fragments, albeit potentially noisy, in shock tubes. Such measurements can provide additional validation for HyChem if rate 
information can be extracted. Moreover, these measurements also carry information about the dependence of individual fragments' chemistries on key parameters that control combustion, including pressure and temperature effects. Such information can also identify how the coupling between the various fragments' chemistries occurs at different conditions.

In the present study, we propose an approach in which time-history measurements of species are used directly to extract rates of reactions of fragments by fitting their temporal profiles with artificial neural networks (ANNs). Subsequently, rates of reactions of fragments, extracted from the slopes of the temporal profiles, are correlated with a subset of the fragments' concentrations using another ANN. While extracting fragments' reaction rates from temporal measurements is novel within the scope of the present study, the tabulation of the fragments' reaction rates in terms of transported temperature and species concentrations is already an established method [12-25]. ANN tabulation offers the potential for reduction in computational memory and speed compared to the use of look-up tables in addition to its ability to model a wide range of data sources [12-14].

We demonstrate our approach using $n$-dodecane as a representative large carbon number fuel, which exhibits the two distinct stages of pyrolysis and oxidation, and we generate 'experimental' species time-history data by imposing synthetic noise to the simulated profiles from a detailed chemistry simulation. The proposed methodology of handling experimental uncertainty in the measurements of species temporal profiles and the construction of the pyrolysis chemistry can be extended to other complex fuels with available experimental data. Such data are becoming increasingly available in the combustion literature (see for example Refs. [26-29]).

\section{ANN Chemistry Reduction Approach}

The present effort addresses two key challenges in handling experimental data with measurement noise towards the assessment of adequate models for pyrolysis and subsequent oxidation. The first challenge concerns the capture of accurate time evolution of species, primarily pyrolysis fragments, during 
the pyrolysis stage. We seek to develop individual fits to temporal profiles of species evolutions in the presence of large experimental noise to evaluate, eventually, their chemical reaction rates. The second challenge is to model the pyrolysis chemistry. In the HyChem approach [5-11], this is implemented by specifying global reactions whose coefficients are fitted through an optimization scheme based on experimental data. These global reactions, as previously adopted for other contexts, are designed to fit the available data. They are not based on lumped elementary reactions; yet, they can be used to establish important scaling relations, such as the dependence of the fragments' chemistries on pressure or the fuel concentration. In the present study, we use the extracted rates from the temporal profiles of fragments' concentrations to develop a regression of these reactions rates in terms of measured concentrations (or temperature if that information is available). To address the above-stated challenges, we rely on artificial neural networks (ANN) for regression of both temporal profiles of fragments' concentrations and for relating the fragments' reaction rates to the fragments' concentrations.

As in HyChem, we adopt a hybrid chemistry approach that consists of ANN-based reaction rates of the fuel fragments and foundational $\mathrm{C}_{0}-\mathrm{C}_{4}$ chemistry for the fragments' oxidation. The process for determining the ANN-based reaction rates is illustrated in Fig. 1 and is summarized as follows:

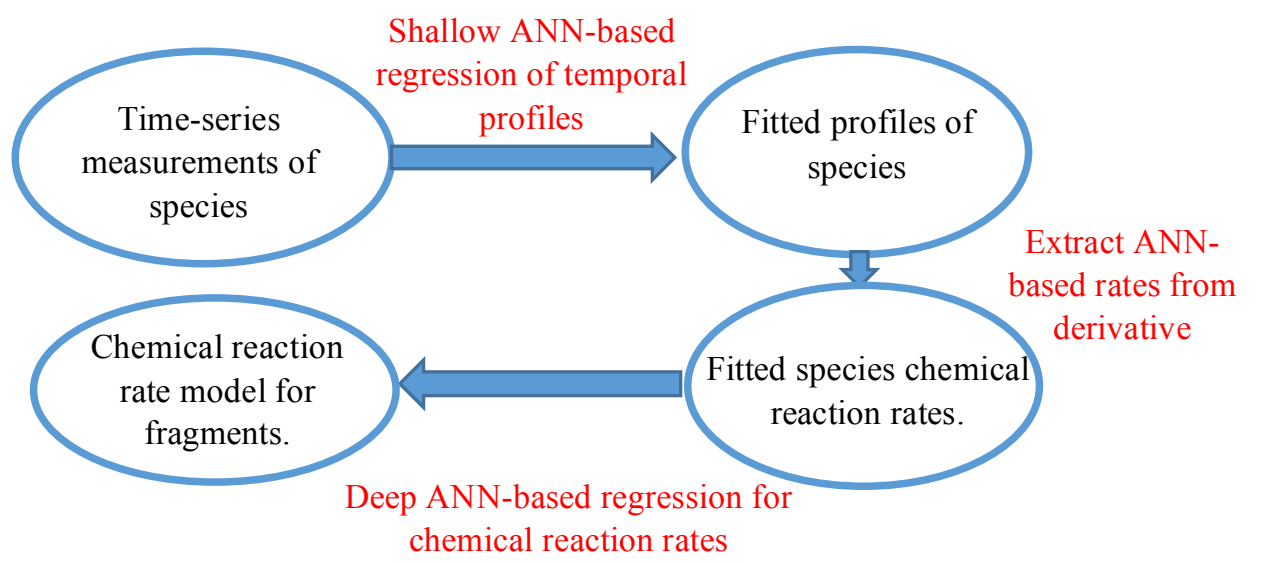

Figure 1. Schematic of the chemistry reduction process for fuel fragments during pyrolysis.

- Starting with raw data for time series measurements of fragments at different conditions of pressure and temperature, a shallow ANN regression is carried out on every measurement individually to obtain 
fitted profiles of the temporal evolution of the species concentrations. A shallow ANN uses a single hidden layer with a few neurons in this layer to capture the trends of the relatively noisy data of the time series measurements.

- A temporal derivative of the ANN functional form for the individual profiles is used to determine the slope of the species, resulting in an evaluation of their reaction rates. Therefore, in contrast to HyChem, we do not explicitly write global reactions or state the corresponding reactants and products in these reactions. Yet, both models are designed to predict the key fragments' reaction rates during the pyrolysis stage.

- The fitted species profiles are related to their corresponding rates using a deep ANN regression, thus providing a reaction rate model for the fuel fragments during the pyrolysis stage. A deep ANN can accommodate more hidden layers and a larger number of neurons per hidden layers compared to the shallow ANNs to able an adequate regression for the species reaction rates.

Finally, the ANN-based chemical reaction rate model is combined with a $\mathrm{C}_{0}-\mathrm{C}_{4}$ foundational chemistry for the fragments' oxidation to form a complete model for the pyrolysis and oxidation of the fuel, as illustrated in Fig. 2. The species not included in the ANN framework are modeled by a $\mathrm{C}_{0}-\mathrm{C}_{4}$ chemical mechanism during the pyrolysis stage and beyond. Therefore, the proposed model can handle oxidation as soon as fragments are produced.

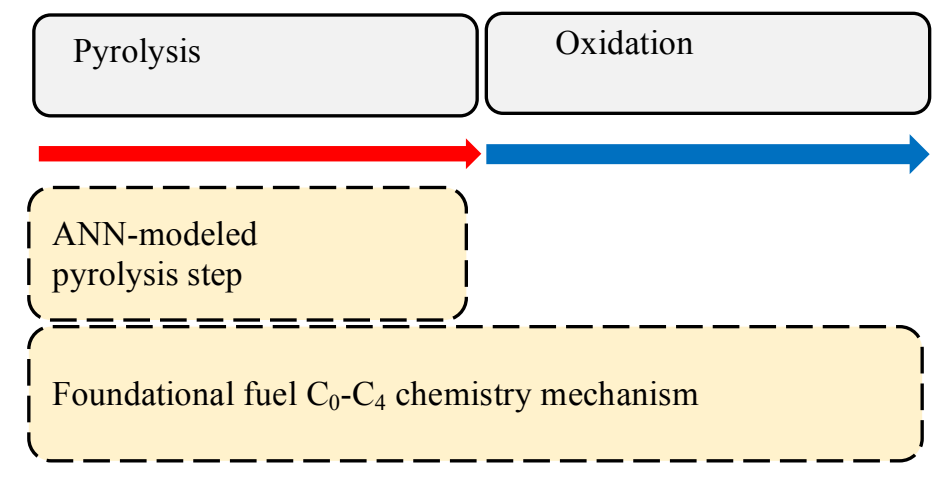

Figure 2. Reduced chemistry model for large hydrocarbon oxidation. 
ANNs are constructs that relate input quantities (for example, for the fitting of temporal profiles, these inputs are time) and output quantities (for example, temporal measurement of fragments' concentrations) through a network of nodes or neurons organized within layers - an input layer, a set of hidden layers and an output layer, that relate the input and the output quantities. Nodes within a given layer can receive 'signals' from nodes from a previous layer and transmit them from a subsequent layer with different weights associated with every connection, as shown in Fig. 3. The connections between various nodes represent the weights used in the final functional form. Hence, a larger number of neurons or hidden layers in a network would directly correspond to a more complex model. The graphical form illustrated in Fig. 3 also has a mathematical form that relates input and output quantities.

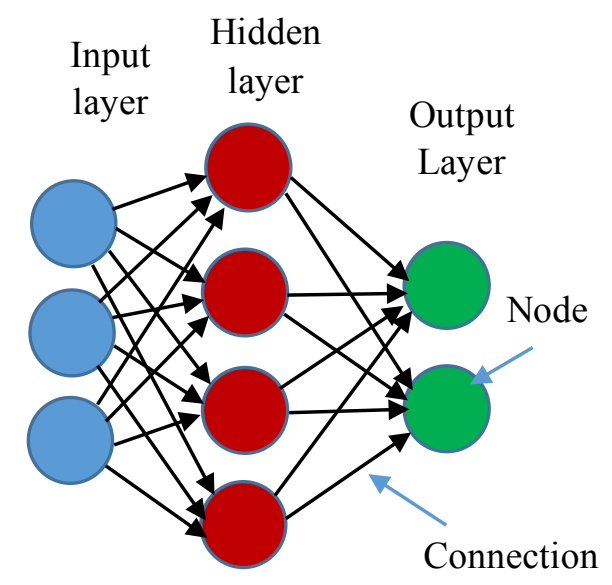

Figure 3. Schematic of an ANN with one input layer of 3 nodes, one hidden layer with 4 nodes and an output layer with 2 nodes.

For example, for experimental measurements involving species profiles vs. time, an output variable $\phi$ (e.g. species concentration) can be expressed as a function for a single hidden layer as:

$$
\phi(t)=W L_{\phi k} f\left(W I_{k} t+B I_{k}\right)+B L_{\phi}
$$

In Eq. (1), $t$ is time (input), $k$ is the number of neurons in the hidden layer; $W I_{k}$ and $B I_{k}$ are the ANN weight matrix and the bias for the initial layer; $W L_{\phi k}$ and $B L_{\phi}$ are the weight matrix and the bias for the output layer; $f$ is the activation function, which corresponds, in our analysis, to the tangent sigmoid function. A principal goal of ANN training is to determine the weight of the connections from the input 
layer all the way to the output layer, thus, establishing a mathematical model of the outputs in terms of the inputs. For tabulation purposes, theses weights and bias values are stored; therefore, regardless of the size of the network, memory allocations are reduced by adopting ANN vs. alternative multi-dimensional tabulation procedures.

From Eq. (1), we can derive an expression for the measured species' reaction rates $S_{\phi}$ of pyrolysis species using the weights from the temporal profile fit as follows:

$$
S_{\phi}=\frac{d \phi}{d t}=W L_{\phi k} \frac{d f}{d u_{k}} \frac{d u_{k}}{d t}, \text { where } u_{k}=W I_{k} t+B I_{k} \text { and } \frac{d u_{k}}{d t}=W I_{k}
$$

Within the context of this work, shallow ANNs are used to establish smooth fits amidst widely fluctuating experimental time-history measurements. These ANNs rely on a single hidden layer with very few neurons. Since the numbers of neurons and hidden layers in a network directly correspond to the number of weight parameters of the mathematical form (2), it is essential to use a smaller number of hidden layers and neurons per hidden layer to track global trends and avoid over-fitting where species concentrations can be well-captured for the exact measurement times and poorly captured in between. For the results presented below, the network architecture requirements for the shallow and deep ANNs were consistent among the different mixtures. For the shallow ANN, one single hidden layer with 2 to 3 neurons with a single input indicating time and a single output for the concentration is used. For the deep ANNs, although they can accommodate more than one hidden layer, we have found that one hidden layer was enough to obtain an acceptable regression for the species chemical reaction terms. However, the number neurons in these hidden layers is much larger than in the case of the shallow ANNs resulting in approximately 40 neurons in the hidden layer.

Figure 4 shows shallow ANN-fitted temporal evolution profiles for 4 fragments $\left(\mathrm{C}_{2} \mathrm{H}_{4}, \mathrm{C}_{3} \mathrm{H}_{6}, i\right.$ $\mathrm{C}_{4} \mathrm{H}_{8}, \mathrm{CH}_{4}$ ) of the pyrolysis of $0.8 \% \mathrm{Cl}$ fuel/argon at $1071 \mathrm{~K}$ and $0.485 \mathrm{~atm}$, based on the data provided in [28]. This fuel is comprised of approximately $75 \%$ iso- $\mathrm{C}_{12} \mathrm{H}_{26}$ and $25 \%$ iso- $\mathrm{C}_{16} \mathrm{H}_{34}$. It may be observed 
that the shallow ANN fits track the experimental data and remove high frequency fluctuations reasonably well. The shallow ANN used to fit the temporal data has one hidden layer and uses only 5 neurons.

(a)

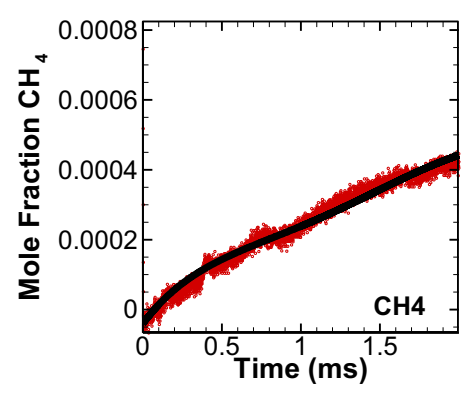

(c)

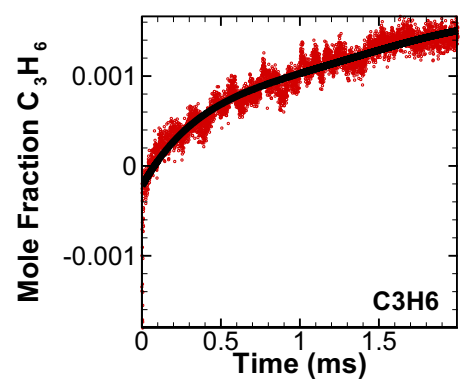

(b)

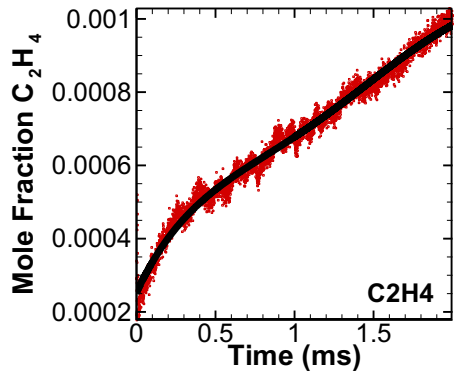

(d)

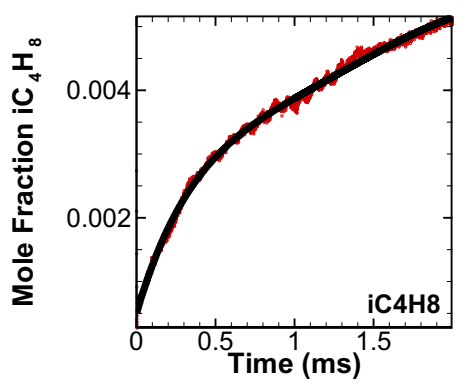

Figure 4. Shallow ANN-based regression of time series of 4 fragments based on the experimental data [28]. Experiment: red symbols; ANN regression: solid black lines.

To verify our approach over wide ranges of conditions, we will employ simulated time evolutions of species using a detailed chemical model with added noise. While the experimental noise in [28] is sufficiently low, it is useful to explore effects of higher experimental error and test a wider array of experimental measurements where the signal-to-noise ratio may be lower than that observed in [28]. It is equally important to explore strategies to improve these fits. Here, we consider the effects of repeating the same measurements as a strategy to improve the regression for reaction rates from noisy temporal profiles.

The smooth and continuous profile provided by shallow ANN fitting can be differentiated to compute smooth reaction rates of fuel fragments. The same fitting strategy can be employed for timehistory measurements ranging over different conditions of initial pressure and temperature. The species' concentration and reaction rates over this broader range of conditions can then be used to build a so-called deep ANN-based reaction rate model representing the chemistry of fuel fragments in the pyrolysis region. 
A deep ANN is different from shallow ANN in the complexity of its network architecture. Typically, a deep ANN contains multiple hidden layers with several neurons per layer.

The next step in the framework is deep ANN tabulation of extracted rates using multiple hidden layers. The objective here is to develop a reaction rate model for the reaction rates of the fuel fragments given their concentrations. As discussed earlier, these reaction rates are already determined from the shallow ANN regression of the fragments' concentrations vs. time. The reaction rates can thus be represented as follows:

$$
\mathbf{S}=\mathbf{f}\left(C_{1}, \quad, C_{\mathrm{N}_{\text {measured }}} ; p, T\right)
$$

where, $\mathbf{S}$ is reaction rates' vector for the measured quantities, which includes species compositions (e.g., given as concentrations, $\left.\mathrm{C}_{1}, \mathrm{C}_{2}, \ldots . \mathrm{C}_{\mathrm{N}}\right)$ and $N_{\text {measured }}$ is the number of measured fuel fragments. The deep ANN regression is designed to develop an accurate model for the measured fuel fragments reaction rates vs. their concentrations during the pyrolysis stage. This model along with the foundational $\mathrm{C}_{0}-\mathrm{C}_{4}$ chemical mechanism can be used to predict the high-temperature oxidation chemistry of the fuel (as illustrated in Fig. 2). Since pyrolysis reaction rates of fuel fragments vary with pressure and temperature, the inputs to the deep ANN may include these important thermo-chemical parameters. It is important to emphasize that the determination of the needed parameters in HyChem also amounts to a global regression for the various reaction rate parameters in the global reactions for the fragments based on an array of temporal and yield measurements for the fragments.

In Eq. (3), a relation between species and their chemical reaction rates is established in composition space in the same spirit of global or detailed chemical kinetics. The complexity of function $\mathbf{f}$ in Eq. (3) provides a capability to handle large non-linearity. The objective of neural network training is to minimize the normalized mean squared error (NMSE) between predicted and original data and, in the process, find 
the best set of weight parameters for $\mathbf{f}$ that can achieve this. In this work, an adaptive algorithm is used to determine the optimum number of neurons and hidden layers that minimize NMSE.

Starting from smooth concentration and reaction rate fits obtained from shallow ANNs for various experimental measurements, this algorithm trains $60 \%$ of the combined data and reserves the remaining for testing. The optimum set of weights obtained from training are used to predict reaction rates for the test data to compute the NMSE. The training algorithm is implemented in Matlab using the LevenbergMarquardt backpropagation with tangent-sigmoid activation functions for all hidden layers. Initially the training is carried out on a very simple network architecture with a single hidden layer containing 5 neurons. The number of neurons or hidden layers are increased incrementally until the tolerance for the NMSE is satisfied or the maximum number of neurons or hidden layers is reached. In this work, the tolerance for NMSE is specified as $10^{-8}$. Again, the objective here is to determine an optimum network architecture to manage the computational cost and accuracy of the ANN regression and store weights of the resulting function $\mathbf{f}$ shown in Eq. (3).

At this point, it may be useful to state some of the trade-offs between adopting the proposed hybrid chemistry approach presented here vs. the HyChem approach [5-11]. One of the obvious characteristics of HyChem [5-11] is its reliance on the law of mass action (through a set of global reactions) to produce important scaling relations, such as the dependence of fuel pyrolysis reactions on pressure or concentrations. Such scaling relations allow the model to be implemented outside the experimental conditions for which the HyChem model is originally developed (if these scaling relations can be extrapolated beyond these conditions). For example, it is quite common to carry out experiments in shock tubes where the mixture concentration is primarily dominated by a diluent (e.g. Ar or $\mathrm{N}_{2}$ ) (see for example Ref. [5-11]) and this dominance is sustained throughout the experiment. Yet, the scaling of the fragments' reaction rates with the initial fuel concentration can be extracted from the global reactions and implemented for mixtures in which the diluent does not represent the predominant specie. 
In our proposed approach, such scaling relations can be incorporated in the model, especially at the level of building the model for the reaction rates. This can be achieved as follows: 1) first, the shallow ANN is used to construct a database relating fragments' concentrations and their reactions based on the experimental conditions, 2) second, scaling relations are established using this data to augment the database beyond those based on experimental conditions, and 3) finally, the deep ANN regression for reaction rates vs. species concentrations is carried out on this augmented database. In step 2, the scaling relations can be implemented as a power relation as explicitly implemented in HyChem or by adopting other regressions for optimum scaling. Alternatively, the scaling relations based on the original experimental database can be incorporated implicitly if the range of experimental conditions is expanded to include all conditions of interest in the reduced mechanism. This alternative strategy is illustrated in the Results section.

To illustrate the steps outlined above, let us assume that we find that the first fragment reaction rate, $d C_{1} / d t$, scales as powers of fragments 1,2 and 3 concentrations, $C_{1}, C_{2}$ and $C_{3}$ :

$$
\frac{d C_{1}}{d t} \quad C_{1}^{a} C_{2}^{b} C_{3}^{c}
$$

This scaling relations indicates that if $C_{2}$ is increased by a factor of 2 , then the first fragment reaction rate is increased by $2^{b}$. Then, we can augment the database on which the scaling relation (4) is developed by considering factors (e.g. considering factors of $1 / 4,1 / 2,2$ and 4) of $C_{1}, C_{2}$ and $C_{3}$ and evaluating the corresponding reaction rates based on the scaling relation (4). This corresponds to step 2 above. Of course, in practice, scaling of the fragments' global reaction rates with concentrations may or may not be adequately be represented in terms of power relations as in Eq. (4).

An additional characteristic of HyChem is its reliance on a global optimization approach, which also means that the model can be constructed using a variety of experimental measurements (e.g., temporal profiles of species concentrations from shock tubes or fragment yields from flow reactors). While 
strategies for a constrained optimization of the deep ANN for reaction rates can be explored to incorporate additional non-temporal data, the present model is based only on temporal profiles. Non-temporal measurements can consist primarily of species yields at a later stage of the pyrolysis or at a subsequent stage past the pyrolysis and can be measured at different times. The corresponding data can be used to extend the temporal fits of the species concentrations beyond the range of the temporal measurements or incorporated along with the temporal measurements to provide a constrained regression of the temporal profiles or can be incorporated along with the temporal measurements to provide a constrained regression of the measured species temporal profiles.

Yet, these temporal profiles offer several advantages. First, the approach can be implemented for a single set or a limited set of experimental conditions since the reaction rates can be extracted based on a single set of individual fragments' temporal profiles. Therefore, the present model can potentially be used to construct a comprehensive hybrid chemistry model or a model for a narrow range or a single set of experimental conditions. Second, the temporal profiles add an additional level of fine-graininess to the data to explore correlations between pairs of individual fragments. Such correlations can determine the absolute requirements for the number of fragments to be tracked and measured. A generic ANN can admit any number of inputs or outputs. Therefore, we are finding that sometimes the deep ANN regression is more accurate, and the ANN architecture is less complex when we specify a subset of the fragments as inputs and not the entire set given the higher potential experimental uncertainty associated with the measurements of some fragments. 


\section{Results and Discussion}

The purpose of the studies presented here is three-fold: 1) first, we would like to address strategies for improving the temporal regression of concentration profiles and the chemical reaction rates through $a$ priori studies, 2) second, we would like to investigate the feasibility of this approach to interpolate among different set of conditions, such as pressure in the present study, and 3) third, we would like to implement the proposed hybrid chemistry procedure in an a posteriori study, which involves an numerical integration of the species profiles, albeit using a simple canonical problem for the ignition of a homogeneous mixture, to show how the ANN-based procedure can be coupled with $\mathrm{C}_{0}-\mathrm{C}_{4}$ chemistry.

Since experimental time-history measurements of fuel fragments are not available, the above procedure for the generation of ANN-based chemistry tabulations is demonstrated here using homogeneous chemistry calculations for $n$-dodecane, $n-\mathrm{C}_{12} \mathrm{H}_{26}$, as a fuel. The JetSurF 2.0 [30] detailed chemical kinetic model, consisting of 348 species and 2163 reversible reactions, is utilized. Numerical simulations are carried out using the Senkin code [31] for a stoichiometric mixture of $0.1 \% n$ dodecane/argon at a constant pressure of 1 atm and initial temperature of $1300 \mathrm{~K}$. Numerical data are generated for the pyrolysis region only, up to the point where the concentration fuel drops to $0.1 \%$ of its initial concentration. The data are generated at every $0.4 \mu \mathrm{s}$ to mimic the measurement frequency of the experimental data in Ref. [28].

To simulate the uncertainty in experimental data, noise is added to the temporal profiles of the measured fragments. This noise is modeled by adding fluctuations from a uniform random distribution having peaks of $30 \%$ of the maximum concentration of the measured fragments. From the experimental profiles already discussed, such peak error appears to be an over-estimate of typical values reported in the literature. For a fragment $\phi$, this perturbation can be expressed as:

$$
\phi_{\text {perturbed }}(t)=\phi_{\text {simulated }}(t)+0.3 \times 2 \times(r-0.5) \times \phi_{\max }
$$


where $r$ is a random number from 0 to 1 . Therefore, the relative error established by this model can be very large for fuel fragments throughout the pyrolysis stage.

An a priori validation of the ANN-based regression is carried out for the temporal evolutions of fragments for 1, 2 and 3 realizations. These realizations are established by starting with the smooth temporal species profiles from the JetSurF simulation and applying different perturbations on the species profiles. Therefore, they can be interpreted within the context of experimental measurements as repeating the experiment 1, 2 or 3 times to obtain 3 different sets of temporal profiles for the species.

The a priori validation is established for temporal evolutions up to $400 \mu$ s resulting in approximately 1000 discrete species "measurements" for training the shallow ANN. This period corresponds to the extent of the pyrolysis stage for a stoichiometric mixture of $0.1 \% n$-dodecane/argon at 1 atm and $1300 \mathrm{~K}$. Comparisons are made between shallow ANN-based regression of the fragments' temporal profiles and the perturbed and unperturbed solutions based on the JetSurF 2.0 [30] detailed mechanism. The shallow ANN uses only one hidden layer with 2-3 neurons, and one neuron in the input (for time) and the output layers (for individual fragments' concentrations).

Figures 5 and 6 compare the ANN regression based on the a priori study to the detailed mechanism simulation for $\mathrm{CH}_{4}$ and $\mathrm{C}_{2} \mathrm{H}_{4}$, respectively, corresponding to 1,2 and 3 realizations. Again, different realizations can be interpreted as carrying out the experiment several times to improve the regression of the temporal profiles. The last subfigure (bottom right) compares the error between regression plots of different realizations and detailed chemistry. Since the shallow ANN regression is designed to capture the pyrolysis stage, the profiles are shown only for this stage. From the plots, it is clear that convergence of the fits is established after only a few experimental realizations despite the large magnitude of the prescribed error. Although not shown here, even higher errors (twice as large) can be accommodated by considering more realizations to establish a convergence of the temporal profiles. This convergence is the 
ultimate test of the non-linear regression carried out on the data. Similar temporal observations are made for other fuel fragments not shown here.
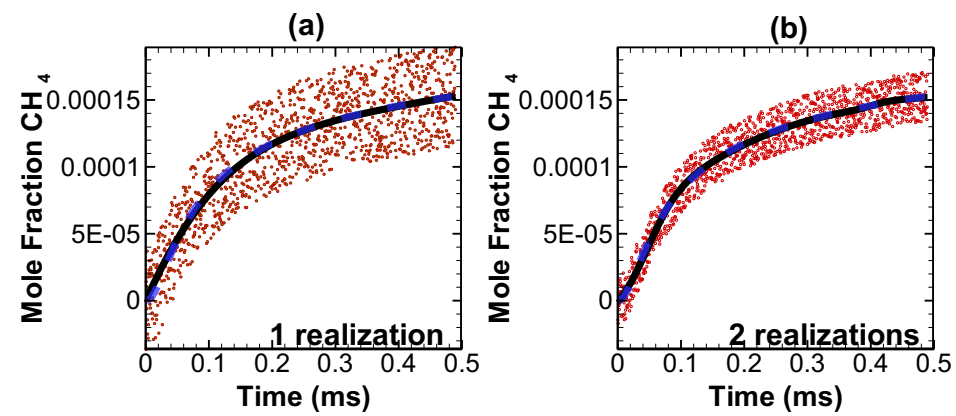

(c)

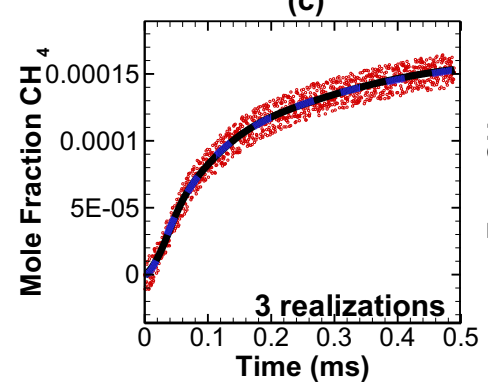

(d)

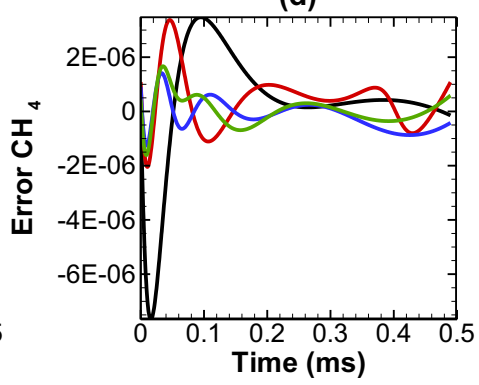

Figure 5. A priori validation of $\mathrm{CH}_{4}$. Shallow ANN (black) vs detailed mechanism (dashed blue) for (a) 1 realization, (b) 2 realizations, (c) 3 realizations, (d) error of ANN fits for 1 (black), 2 (red), 3 (blue) and 5 (green) realizations.
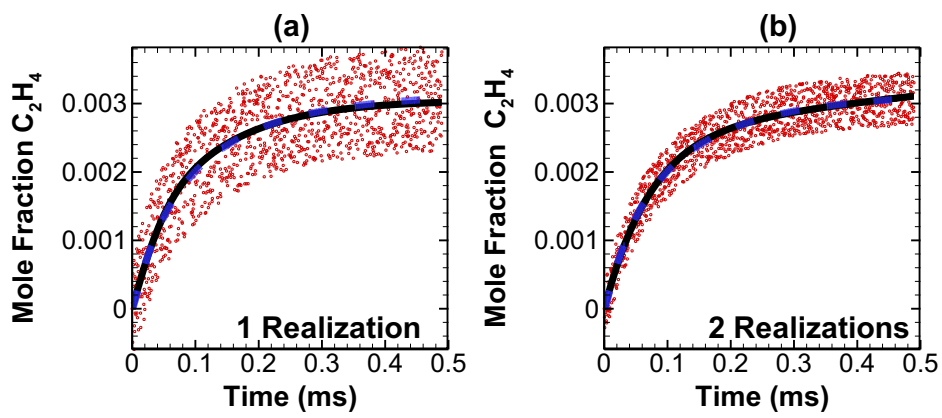

(c)

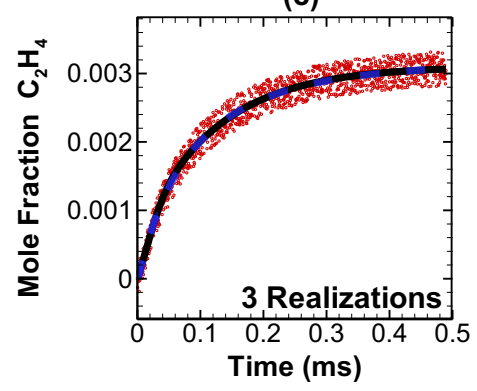

(d)

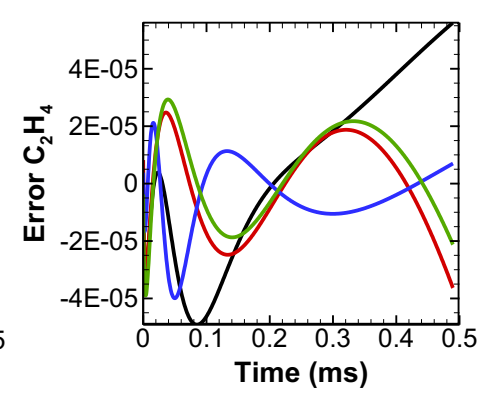

Figure 6. $A$ priori validation of $\mathrm{C}_{2} \mathrm{H}_{4}$. Shallow ANN (black) vs detailed mechanism (dashed blue) for (a) 1 realization, (b) 2 realizations, (c) 3 realizations, (d) error of ANN fits for 1 (black), 2 (red), 3 (blue) and 5 (green) realizations. 
(a)

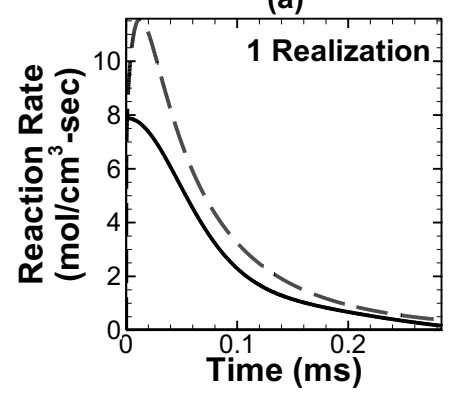

(c)

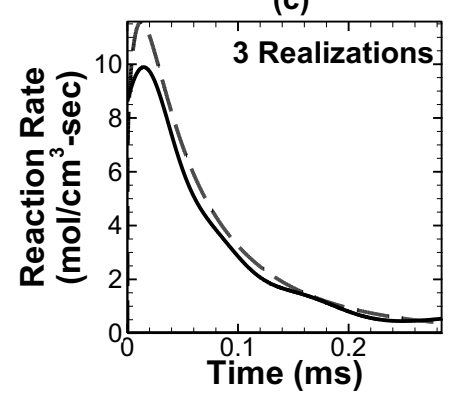

(b)

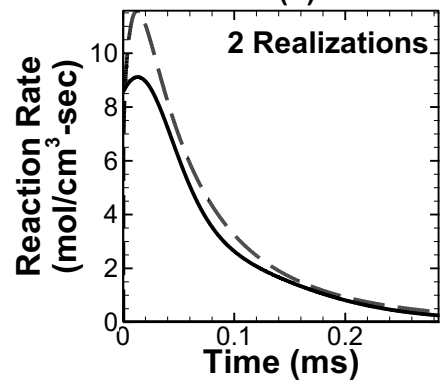

(d)

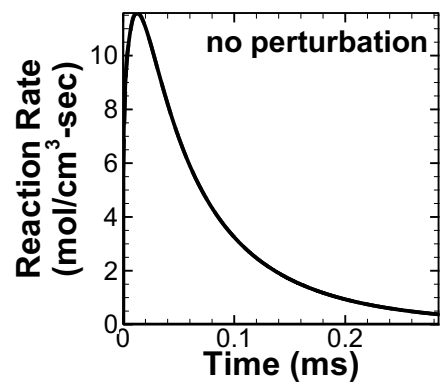

Figure 7. A priori reaction rate comparison of $\mathrm{C}_{2} \mathrm{H}_{4}$. Shallow ANN (solid) vs detailed mechanism (dashed) for (a) 1 realization, (b) 2 realizations, (c) 3 realizations, (d) no perturbation.

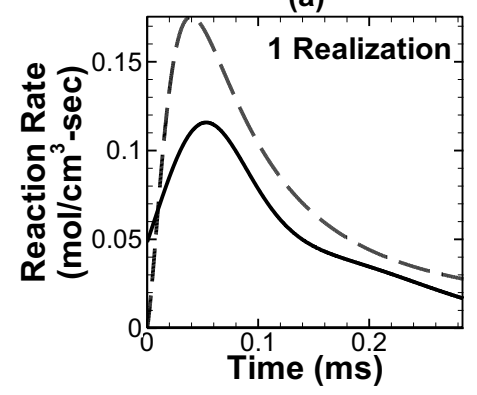

(c)

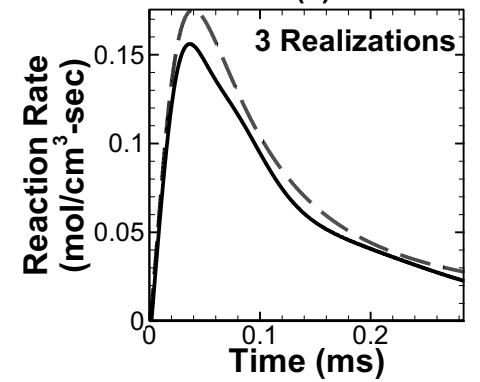

(b)

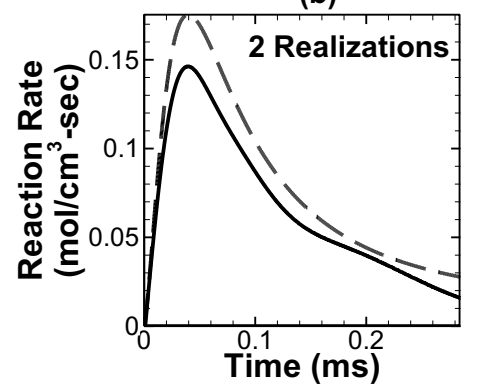

(d)

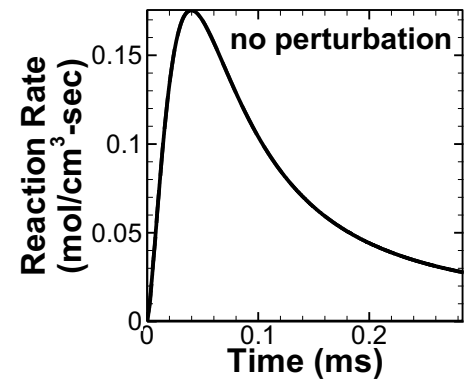

Figure 8. A priori reaction rate comparison of $\mathrm{CH}_{4}$. Shallow ANN (solid) vs detailed mechanism (dashed) for (a) 1 realization, (b) 2 realizations, (c) 3 realizations, (d) no perturbation.

Next, we compare the reaction rates of fuel fragments obtained from shallow ANN and detailed chemistry. The reaction rates for shallow ANNs are computed by differentiating the smooth fits with 
respect to time (see Eq. (2)). The reaction rates for $\mathrm{CH}_{4}$ and $\mathrm{C}_{2} \mathrm{H}_{4}$ obtained for 1,2 and 3 realizations are compared with reaction rates from JetSurF 2.0 [30] in Figs. 7 and 8. Additionally, reaction rates obtained from a shallow ANN based on unperturbed numerical data also are presented to validate the shallow ANN method for reaction rate computation.

We can observe from Fig. 7 and Fig. 8 that the shallow ANN predictions for reaction rates are reasonably close to those predicted by JetSurF 2.0 [30] and the reaction rate predictions for $\mathrm{CH}_{4}$ and $\mathrm{C}_{2} \mathrm{H}_{4}$ improve as the number of realizations are increased. Hence, the ANN time-series fits can be improved by simply increasing the number of realizations. Considering the trade-off between experimental cost and number of realizations, a maximum of 3 realizations are tested here. Moreover, if the perturbations are removed, then the reaction rates match exactly with those of JetSurF 2.0 [30] indicating that any difference in reaction rates is a result of the heavy perturbations induced in the data or the constraint on the experimental data measurement frequency and cannot be attributed to the shallow ANN methodology.

Next, we attempt to gauge the validity of this procedure over a broader range of thermo-chemical compositions. Here, we investigate whether relying on a limited set of measurements at 2 pressure conditions can be used to evaluate the fragments' chemical reaction rates at intermediate conditions between these pressures. In this study, we have generated time-history data of key fragments in the pyrolysis region using the JetSurF 2.0 mechanism [30] for a stoichiometric mixture of $0.1 \% n$ dodecane/argon at $1300 \mathrm{~K}$ at two pressures, 1 and $16 \mathrm{~atm}$. The numerical data are synthetically perturbed using Eq. (4) and a reaction rate model is developed following the procedure described in Sec. 2 using 2 realizations. The resulting hybrid chemistry model is used to predict the evolution of a homogeneous mixture with the same composition and temperature at intermediate pressures, 2, 4, and 8 atm; and these predictions are compared to detailed chemistry calculations using JetSurF 2.0 [30]. The comparisons are shown in Fig. 9. 
It may be observed from Fig. 9 that the reaction rate predictions of the ANN-based rate model match reasonably well those based on the detailed chemical mechanism. Although, the ANN-based model is calibrated for a limited number of experimental measurements made at initial pressures of 1 and $16 \mathrm{~atm}$, it can effectively capture a broader range of conditions in the pyrolysis region.

At this point, we have demonstrated some important aspects of the proposed ANN-based hybrid chemistry model ability to extract reasonably accurate reaction rates from fluctuating time-history data of fuel fragments. With increasing realizations, the fits for fuel fragment concentrations and reaction rates converge toward the detailed mechanism predictions. Moreover, the model is capable of interpolating between broader thermo-chemical states without requiring many experimental measurements, thereby implicitly incorporating the scaling relations useful for predictions over a broader range.
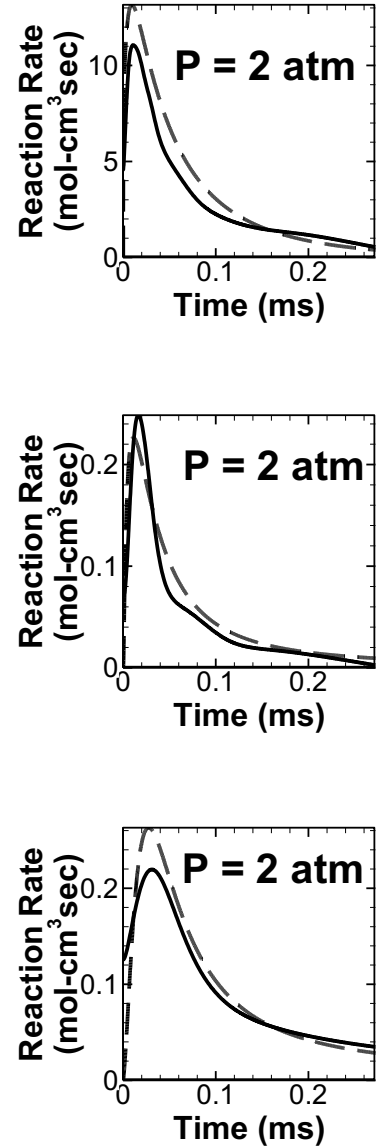

A

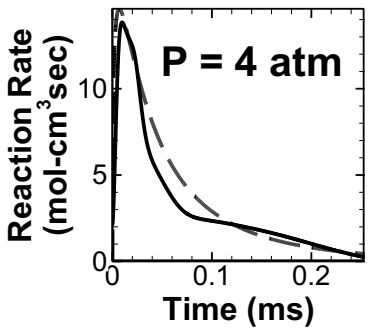

B

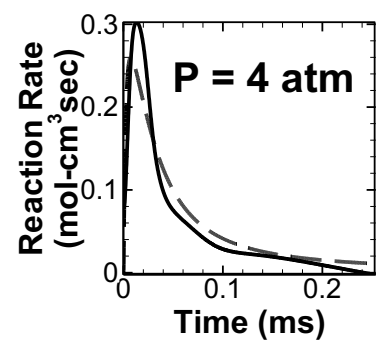

C

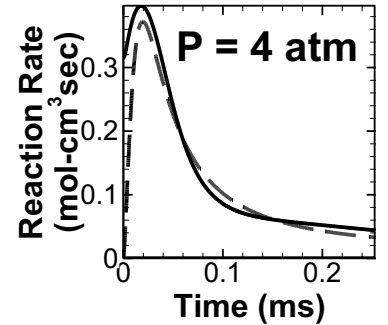

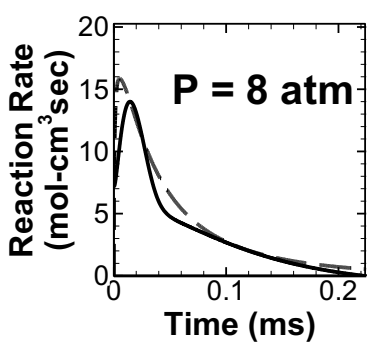
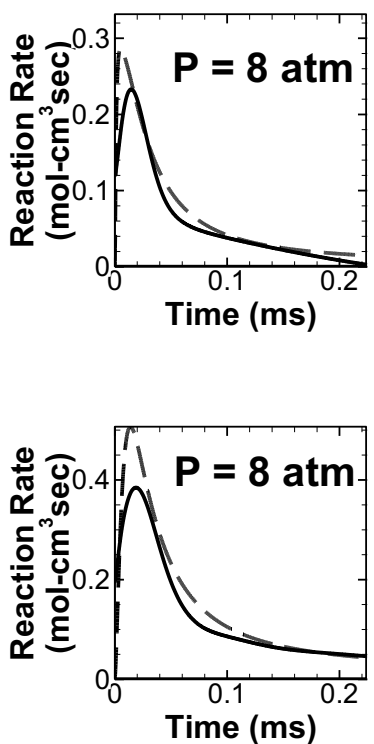

Figure 9. Reaction rate comparisons of $\mathrm{ANN}$-based model (solid) and detailed mechanism (dashed) at 2, 4 and $8 \mathrm{~atm}$ for fuel fragments, (A) $\mathrm{C}_{2} \mathrm{H}_{4}$, (B) $\mathrm{H}_{2}$ and (C) $\mathrm{CH}_{4}$. 
Next, an a posteriori analysis is carried out for the pyrolysis and oxidation stages to validate the ANN-based hybrid chemistry approach adopted here. This test is essential to gauge if the ANN-based reaction rate model representing the fuel fragment chemistry in the pyrolysis region blends well with the foundational $\mathrm{C}_{0}-\mathrm{C}_{4}$ chemistry and yields expected results in terms of species' concentration and temperature profiles. The calculations now involve, as illustrated in Fig. 2, 1) the integration of the fuel fragments chemistry with the ANN-based regression for the chemical reaction rates during the pyrolysis stage and subsequently using foundational $\mathrm{C}_{0}-\mathrm{C}_{4}$ chemistry during the oxidation stage, and 2) the integration of the remaining species using the foundational $\mathrm{C}_{0}-\mathrm{C}_{4}$ chemistry throughout. Here, the foundational $\mathrm{C}_{0}-\mathrm{C}_{4}$ chemistry is based on the USC-Mech II mechanism [32] with 111 species (including the measured fragments) and 784 reversible reactions. The ANN-based pyrolysis reactions are used in the simulation until the concentration of a key fuel fragment, $\mathrm{C}_{2} \mathrm{H}_{4}$, reaches $99 \%$ of its maximum value. Here, $\mathrm{C}_{2} \mathrm{H}_{4}$ is chosen to assist the transition from pyrolysis to oxidation because it has the highest peak concentration among rest of the fragments. The solution is integrated to $20 \mathrm{~ms}$, which incorporates the shorter pyrolysis stage along with a good portion of the fragments' oxidation stage. The pyrolysis stage lasting only a few hundred micro-seconds is a relatively small fraction of this entire integration time. Therefore, the clear computational saving is associated with the integration of the USC-Mech II mechanism with 111 species and 784 reversible reactions compared to the integration with the full JetSurF 2.0 mechanism with 348 species and 2163 reversible reactions, resulting in a three-fold speed up in the computational time. Of course, while a speed up of the solution is important, the real advantage of the present model and that of HyChem is the ability to model the oxidation of a complex fuel when no mechanism is available to describe this oxidation.

In the following discussion, we present 3 different a posteriori cases that identify the potential contributions of omitting key thermo-chemical variables from measurements and to assess the role of measurement uncertainty. One important variable is temperature, which we consider in our simulated 
experiment, but it is generally not accessible in shock tube measurements. The temperature, for the case considered, undergoes a drop during the pyrolysis stage due to endothermic reactions accompanying fuel pyrolysis. This drop is associated with the pyrolysis chemistry and may not be captured by oxidation steps modeled by the foundational $\mathrm{C}_{0}-\mathrm{C}_{4}$ chemistry. The 3 a posteriori cases considered are summarized below:

- The first case, case A corresponds to: 1) no simulated experimental error, 2) the measurement of 8 fragments identified by Wang and co-workers [5-11], which include: $\mathrm{C}_{2} \mathrm{H}_{4}, \mathrm{C}_{3} \mathrm{H}_{6}, 1-\mathrm{C}_{4} \mathrm{H}_{8}, \mathrm{CH}_{4}, \mathrm{H}_{2}, i$ $\mathrm{C}_{4} \mathrm{H}_{8}, \mathrm{C}_{6} \mathrm{H}_{5} \mathrm{CH}_{3}$ and $\mathrm{C}_{6} \mathrm{H}_{6}$, and 3) the inclusion of temperature as a measured quantity. Accordingly, ANN regression for the heat release contribution to temperature is implemented using the same approach. It is to be noted that this is the best-case scenario, since the shallow ANN is fitted on unperturbed data, and temperature and 8 fuel fragments are considered in the reaction rate model. Results of this case are presented in Fig. 10.
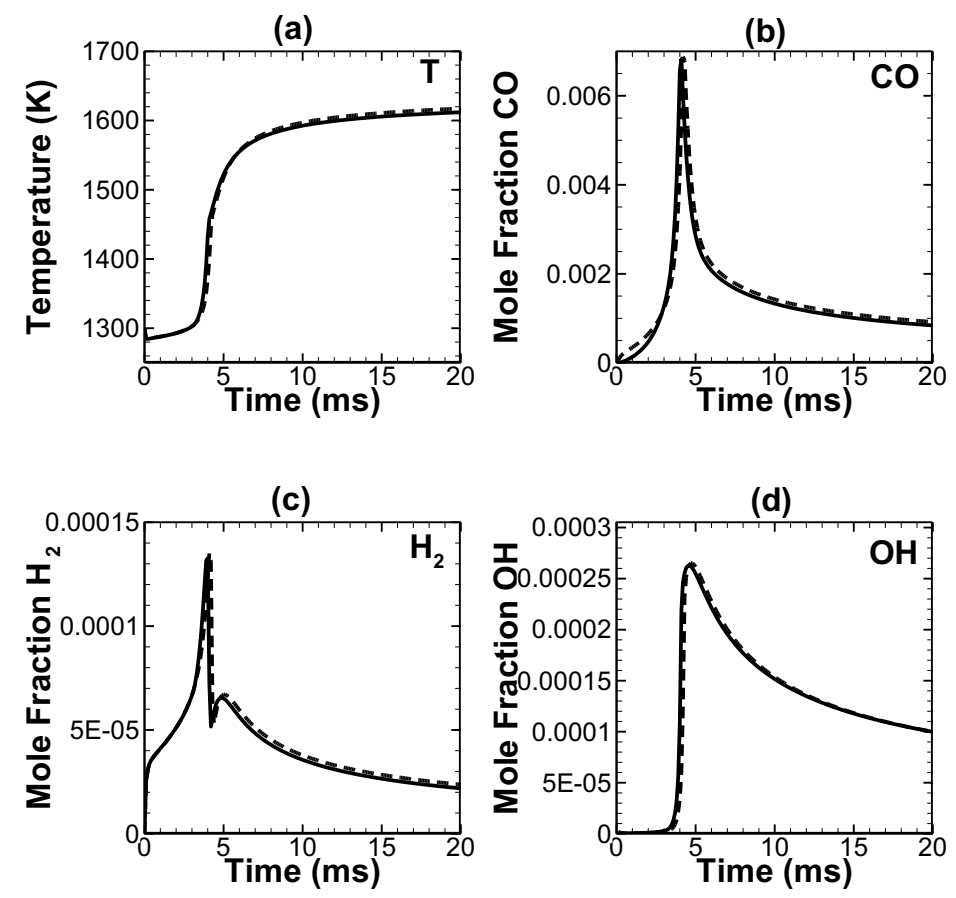

Figure 10. A posteriori comparison of $\mathrm{CO}, \mathrm{H}_{2}$ and $\mathrm{OH}$ mole fractions. Case A (solid); detailed mechanism (dashed).

- The second case, case B, differs from case A by excluding temperature from measurements. Therefore, temperature is modeled using chemical reaction rates from the foundational $\mathrm{C}_{0}-\mathrm{C}_{4}$ chemistry alone. The 
idea here is to gauge the effect of temperature on ANN-based reaction rate modeled during pyrolysis. Results of this case are presented in Fig. 11.

- The third case, case C, differs from case B in 2 aspects. Now, we simulate experimental error of $30 \%$, as described above, and construct the temporal fits for the fragments using 2 realizations. Moreover, we only consider 6 measured fragments, which include: $\mathrm{C}_{2} \mathrm{H}_{4}, \mathrm{C}_{3} \mathrm{H}_{6}, 1-\mathrm{C}_{4} \mathrm{H}_{8}, \mathrm{CH}_{4}, \mathrm{H}_{2}$ and $i-\mathrm{C}_{4} \mathrm{H}_{8}$. The remaining fragments are eliminated since their concentrations are smaller and have negligible impact on the final solution. Additionally, like case B, temperature is excluded from measurements. This case is designed to provide a very stringent test for the proposed ANN-based framework. Results of this case are presented in Fig. 12.
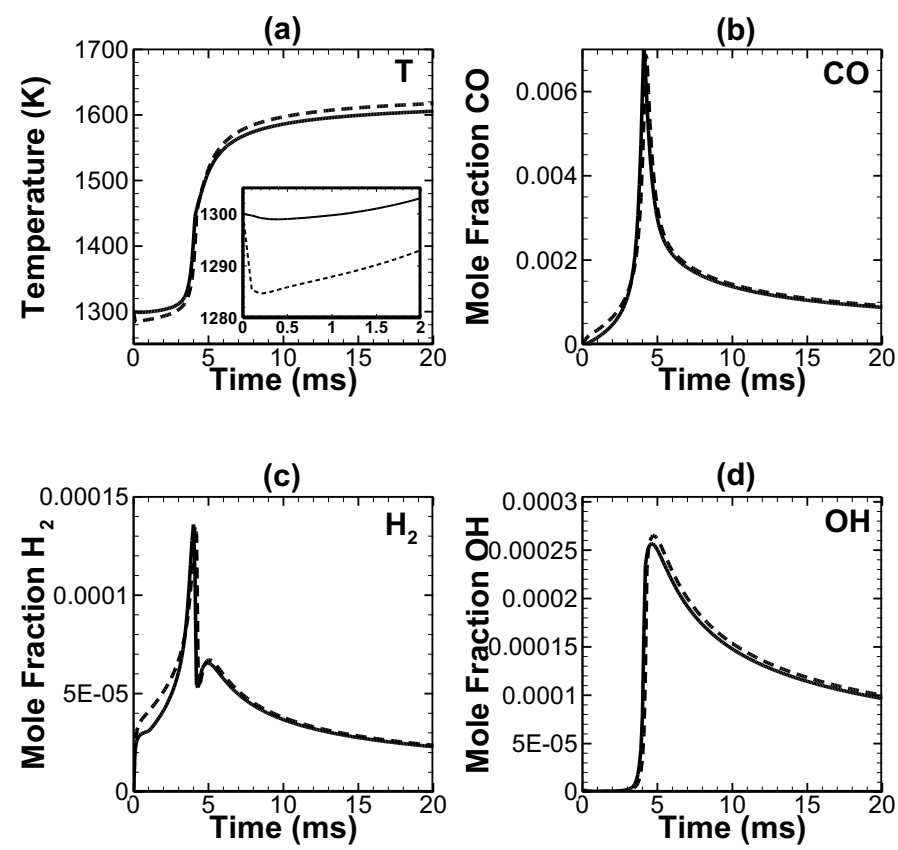

Figure 11. A posteriori comparison of temperature (a), $\mathrm{CO}(\mathrm{b}), \mathrm{H}_{2}$ (c) and $\mathrm{OH}(\mathrm{d})$ mole fractions and temperature over a time range of $500 \mu \mathrm{s}$ (e). Case B (solid); detailed mechanism (dashed).

Figure 11 shows the temporal evolution of temperature, $\mathrm{CO}, \mathrm{H}_{2}$ and $\mathrm{OH}$ mole fractions based on case A for the same initial conditions described in Figs. 5 and 6 and comparing the integration of the chemistry using the ANN-based hybrid scheme (solid lines) and the detailed mechanism based on JetSurF 2.0 [30] (dashed lines). Without experimental error, by including temperature as a measured quantity and by measuring 8 fragments, the comparison is designed to evaluate the accuracy of the ANN-based 
chemistry tabulation. The agreement is very good, and any minor deviations may be attributed to the small error in coupling pyrolysis and oxidation stages. Nonetheless, errors associated with this coupling can still be considered negligible given the performance of the ANN-based hybrid chemistry model.

Figure 11 shows the temporal evolution of temperature, $\mathrm{CO}, \mathrm{H}_{2}$ and $\mathrm{OH}$ mole fractions based on case B for the same initial conditions described in case A. The integration of the chemistry using the ANNbased hybrid scheme (solid lines) is compared with the detailed mechanism based on JetSurF 2.0 [30] (dashed lines). The temperature profiles also are shown for a narrow range to $2 \mathrm{~ms}$ in Fig. 11a. Here, the main difference between case A and case B is the exclusion of temperature as a measured quantity, such that its corresponding heat release term is represented entirely by USC Mech II [32]. The agreement is good for the species; but the final temperature is off by $8 \mathrm{~K}$. The detailed mechanism results in a slight temperature drop, as shown in the inset of Fig. 11a, during the first milliseconds, which is associated with endothermic reactions in this mechanism. This drop is not captured by the simpler foundational chemistry [32] compared to JetSurF 2.0 [30], which incorporates the chemistry of larger hydrocarbons and the fuel. This error propagates and results in a deviation in the final temperature. Nonetheless, the comparison indicates that the hybrid chemistry and detailed chemistry profiles match reasonably well, even without the availability of temperature measurements.

Figure 12 compares a posteriori temporal profiles based on case $\mathrm{C}$ for temperature and three key intermediates of the oxidation process, $\mathrm{CO}, \mathrm{H}_{2}$ and $\mathrm{OH}$ (solid lines) and the detailed chemistry calculations based on JetSurF 2.0 [30] (dashed lines). This case is designed to provide a very stringent test for the proposed ANN-based framework. The results show that the ANN-based hybrid chemistry approach tracks reasonably well the detailed chemistry results despite additional constraints imposed in case C. Although not clearly shown in the figure, the final temperature based on the present hybrid chemistry approach is off by $8 \mathrm{~K}$. Again, this difference may be attributed to the role of endothermic reactions during the pyrolysis stage. Strategies to address this difference will be considered as an additional refinement of the 
proposed hybrid chemistry model. Despite the use of only 2 realizations/experiments, this small number of realizations appears to be adequate for the present comparisons. Since hydroxyl is the main chain branching radical at high temperatures, an accurate prediction of the $\mathrm{OH}$ concentration and its rise time establishes that the ANN-based approach can adequately capture the auto-ignition of a large fuel such as $n$-dodecane. This observation is further illustrated in the following discussion.
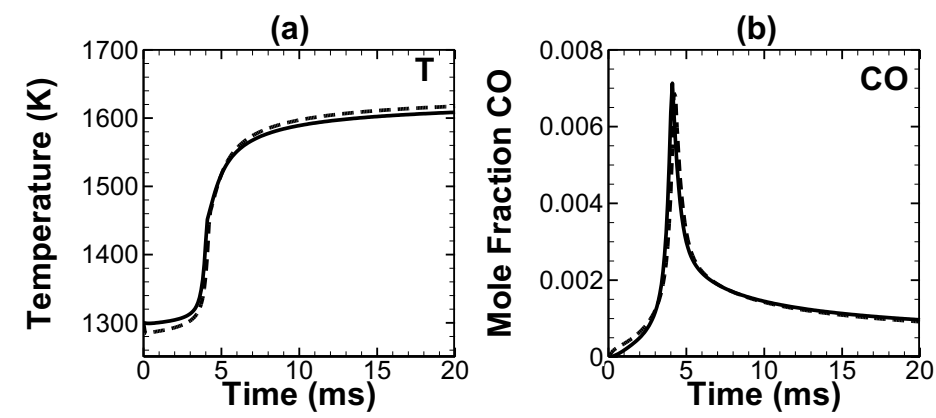

(c)
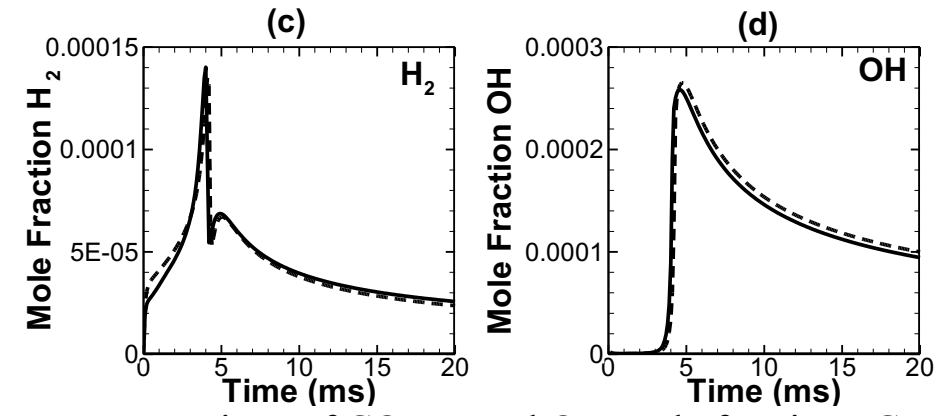

Figure 12. A posteriori comparison of $\mathrm{CO}, \mathrm{H}_{2}$ and $\mathrm{OH}$ mole fractions. Case $\mathrm{C}$ (solid); detailed mechanism (dashed).

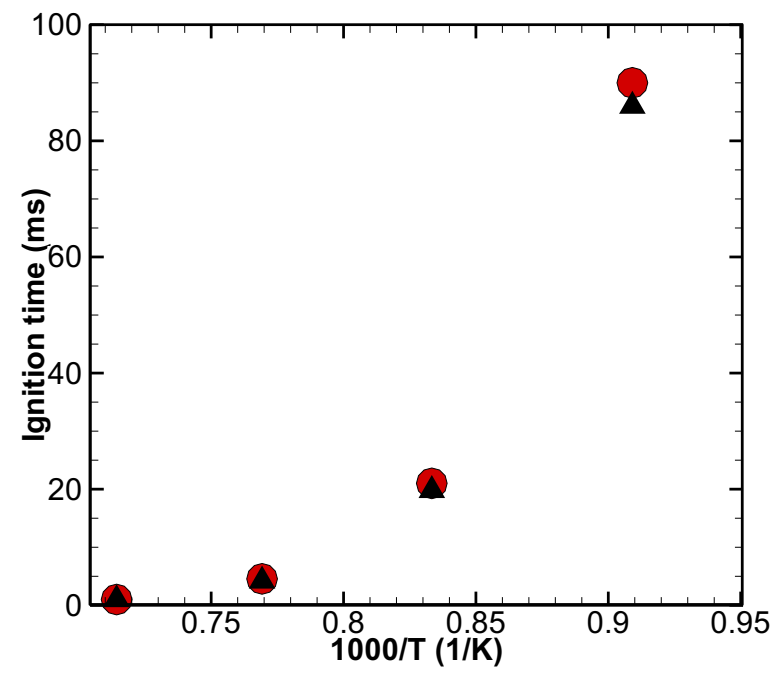

Figure 13. A posteriori validation comparing the proposed ANN based hybrid chemistry (filled red circles) with detailed mechanism (filled black triangles) for ignition delay times at different initial temperatures. 
Next, we investigate our model's ability to predict a global quantity, the ignition delay time. The comparisons are based on case $\mathrm{C}$ presented in Fig. 12, except that, now, the initial temperatures are varied to include 1100,1200 , and $1400 \mathrm{~K}$. The initial pressure and mixture composition are identical to cases A, $\mathrm{B}$ and $\mathrm{C}$. The ignition delay times are based on the peak of $\mathrm{OH}$ concentration; however, other indicators, such as the maximum temperature gradient or the peak mole fraction of $\mathrm{H}$ or $\mathrm{O}$ radicals yield similar values. Figure 13 compares ignition delay times based on case $\mathrm{C}$ (filled red circles) with detailed chemistry simulations using the JetSurF 2.0 chemical mechanism [30] (filled black triangles). A very good agreement between the ANN-based hybrid chemistry and JetSurF 2.0 [30] is obtained, as discussed in relations to the species and temperature profiles in Fig. 12. 


\section{Conclusions}

An ANN-based regression/tabulation framework is proposed for a hybrid chemistry scheme to predict the high-temperature oxidation of large hydrocarbons starting with experimental time series data of pyrolysis products. Moreover, the ANN-based regression for the prediction of fragments' temporal profiles and their reaction rates can be used as a pre-processing step within the context of the HyChem approach proposed by Wang and co-workers [5-11] since it is able to handle species profiles separately and can be improved with the inclusion of additional sets of experimental time-series measurements. This regression step is critical for the proposed framework where reaction rates are derived directly from the temporal profiles. We have established that even with a relatively large measurement noise or error, temporal patterns can be obtained, and improved patterns can converge after a few realizations or experiments. This convergence is a valuable measure of the accuracy of the time fits. A priori and $a$ posteriori analysis of the proposed approach shows that the ANN-based hybrid chemistry approach can yield an effective and accurate chemistry reduction scheme for the pyrolysis stage. Additionally, the reasonably accurate reaction rate predictions over a broader range of conditions indicate the applicability of this approach to handle a broader range of initial temperature and pressure conditions. Finally, with the availability of actual experimental data the proposed approach can be used to build reduced mechanisms for complex fuels and provide the capability to explore other machine learning strategies like selforganizing maps (SOM) $[19,24,25,33]$ for data clustering and real-time refinement of chemical mechanisms. Other strategies for chemistry reduction will be considered as extensions of the proposed framework based on the two-step procedure discussed here. While potential accuracy and speed up can be explored with a combination of ANN-SOM regression, other regression methods will be explored. For example, a partition of the composition space via SOM can potentially enable less computationally intensive regression methods for the species reaction rates, such as the use of low-order polynomial fits, such as what is implemented in the PRISM model [1]. Functional mapping strategies to advance the 
solution of a chemical system by a small increment in time, such as the ISAT [34] or the PRISM [1] approaches also represent viable extensions to the proposed framework.

\section{Acknowledgements}

Dr. Aamir Farooq would like to thank the Office of Sponsored Research at the King Abdullah University of Science and Technology (KAUST) for financial support. Sultan Alqahtani would like to acknowledge the support of King Khalid University in Abha, Saudi Arabia.

\section{References}

1. T. Turanyi, A.S. Tomlin, Reduction of reaction mechanisms, in Analysis of Kinetic Reaction Mechanisms, pp. 183-312, Springer, 2014.

2. X. You, F.N. Egolfopoulos, H. Wang, Detailed and simplified kinetic models for $n$-dodecane oxidation: The role of fuel cracking in aliphatic hydrocarbon combustion, Proc. Combust. Inst. 32 (2009) 403-410.

3. T.J. Held, A.J. Marchese, F.L. Dryer, A semi-empirical reaction mechanism for n-heptane oxidation and pyrolysis, Combust. Sci. Technol. 123 (1997) 107-146.

4. S.P. Zeppieri, S.D. Klotz, F.L. Dryer, Modeling concepts for larger carbon number alkanes: A partially reduced skeletal mechanism for $n$-decane oxidation and pyrolysis, Proc. Combust. Inst. 28 (2000) $1587-1595$.

5. R. Xu, H. Wang, D.F. Davidson, R.K. Hanson, C.T. Bowman, F.N. Egolfopoulos, Evidence supporting a simplified approach to modeling high-temperature combustion chemistry, $10^{\text {th }}$ U.S. National Combustion Institute Meeting, The Combustion Institute, College Park, MD, April 23-26, 2017.

6. R. Xu, D. Chen, K. Wang, T. Tao, J.K. Shao, T. Parise, Y. Zhu, S. Wang, R. Zhao, D.J. Lee, F.N. Egolfopoulos, D.F. Davidson, R.K. Hanson, C.T. Bowman, H. Wang, HyChem Model: Application 
to petroleum-derived jet fuels, $10^{\text {th }}$ U.S. National Combustion Institute Meeting, The Combustion Institute, College Park, MD, April 23-26, 2017.

7. K. Wang, R. Xu, T. Parise, J.K. Shao, D.J. Lee, A. Movaghar, D.F. Davidson, R.K. Hanson, H. Wang, C.T. Bowman, F.N. Egolfopoulos, Combustion kinetics of conventional and alternative jet fuels using a hybrid chemistry (HyChem) approach, $10^{\text {th }}$ U.S. National Combustion Institute Meeting, The Combustion Institute, College Park, MD, April 23-26, 2017.

8. K. Wang, R. Xu, T. Parise, J.K. Shao, D.F. Davidson, R.K. Hanson, H. Wang, C.T. Bowman, Evaluation of a hybrid chemistry approach for combustion blended petroleum and bio-derived jet fuels, $10^{\text {th }}$ U.S. National Combustion Institute Meeting, The Combustion Institute, College Park, MD, April 23-26, 2017.

9. R. Xu, D. Chen, K. Wang, H. Wang, A comparative study of combustion chemistry of conventional and alternative jet fuels with hybrid chemistry approach, $55^{\text {th }}$ AIAA Aerospace Sciences Meeting, AIAA paper 2017-0607, Grapevine, Texas, January 9-13, 2017.

10. H. Wang, R. Xu, K. Wang, C.T. Bowman, R.K. Hanson, D.F. Davidson, K. Brezinsky, F.N. Egolfopoulos, A physics-based approach to modeling real-fuel combustion chemistry-I. Evidence from experiments, and thermodynamic, chemical kinetic and statistical considerations. Combust. Flame 193 (2018) 502-519.

11. R. Xu, K. Wang, S. Banerjee, J.K. Shao, T. Parise, Y.Y. Zhu, S.K. Wang, A. Movaghar, D.J. Lee, R.H. Zhao, X. Han, Y. Gao, T.F. Lu, K. Brezinsky, F.N. Egolfopoulos, D.F. Davidson, R.K. Hanson, C.T. Bowman, H. Wang, A Physics-based approach to modeling real-fuel combustion chemistry-II. Reaction kinetic models of jet and rocket fuels, Combust. Flame 193 (2018) 520-537.

12. T. Turanyi, A.S. Tomlin, Storage of chemical kinetic information, in Cleaner Combustion, Green Energy and Technology (F. Battin-Leclerc et al. (Eds.)), Springer-Verlag London (2013) pp. 485-512. 
13. F.C. Christo, A.R. Masri, E.M. Nebot, T. Turanyi, Utilising artificial neural network and repromodelling in turbulent combustion, 1995 IEEE Int. Conf. Neural Networks Proc. (1995) 911-916.

14. F.C. Christo, A.R. Masri, E.M. Nebot, S.B. Pope, An integrated PDF/neural network approach for simulating turbulent reacting systems, $26^{\text {th }}$ Symp. (Int.) on Combustion (1996) 43-48.

15. F.C. Christo, A.R. Masri, E.M. Nebot, Artificial neural network implementation of chemistry with pdf simulation of H2/CO2 flames, Combust. Flame 106 (1996) 406-427.

16. J.A. Blasco, N. Fueyo, C. Dopazo, J. Ballester, Modelling the temporal evolution of a reduced combustion chemical system with an artificial neural network, Combust. Flame 113 (1998) 38-52.

17. J.A. Blasco, N. Fueyo, J.C. Larroya, C. Dopazo, J.Y. Chen, A single-step time-integrator of a methaneair chemical system using artificial neural networks, Comput. Chem. Eng. 23 (1999) 1127-1133.

18. J.Y. Chen, J.A. Blasco, N. Fueyo, C. Dopazo, An economical strategy for storage of chemical kinetics: Fitting in situ adaptive tabulation with artificial neural networks, Proc. Combust. Inst. 28 (2000) 115121.

19. J.A. Blasco, N. Fueyo, C. Dopazo, J.Y. Chen, A self-organizing-map approach to chemistry representation in combustion applications, Combust. Theo. Model. (2000) 61-76.

20. M. Ihme, A.L. Marsden, H. Pitsch, Generation of optimal artificial neural networks using a pattern search algorithm: Application to approximation of chemical systems, Neural Comput. 20 (2008) 573601.

21. M. Ihme, C. Schmidt, H. Pitsch, Optimal artificial neural networks and tabulation methods for chemistry representation in LES of a bluff-body swirl-stabilized flame, Proc. Combust. Inst. 32 (2009) 1527.

22. B.A. Sen, S. Menon, Linear eddy mixing based tabulation and artificial neural networks for large eddy simulations of turbulent flames, Combust. Flame 157 (2010) 62-74. 
23. B.A. Sen, S. Menon, Large eddy simulation of extinction and reignition with artificial neural networks based chemical kinetics, Combust. Flame 157 (2010) 566-578.

24. A.K. Chatzopoulos, S. Rigopoulos, A chemistry tabulation approach via rate-controlled constrained equilibrium (RCCE) and artificial neural networks (ANNs), with application to turbulent nonpremixed CH4/H2/N2 flames, Proc. Combust. Inst. 34 (2013) 1465-1473.

25. L.L.C. Franke, A.K. Chatzopoulos, S. Rigopoulos, Tabulation of combustion chemistry via artificial neural networks: Methodology and application to LES-PDF simulation of Sydney flame L, Combust. Flame 185 (2017) 245-260.

26. D. F. Davidson, Z. Hong, G. Pilla, A. Farooq, R. Cook, R. K. Hanson, Multi-species time-history measurements during $n$-dodecane oxidation behind reflected shock waves, Proc. Combust. Inst. 33 (2011) 151-157.

27. D. Haylett, D. F. Davidson, R. Cook, Z. Hong, W. Ren, S. Pyun, R. K. Hanson, Multi-species time history measurements during n-hexadecane oxidation behind reflected shock waves, Proc. Combust. Inst. 34 (2013) 369-376.

28. T.D. Parise, D. F. Davidson, R. K. Hanson, Shock tube/laser absorption measurements of the pyrolysis of a bimodal test fuel, Proc. Combust. Inst. 36 (2017), 281-288.

29. J. Tamour, E.F. Nasir, E. Es-Sebbar, A. Farooq, A comparative study of the oxidation characteristics of two gasoline fuels and an n-heptane/iso-octane surrogate mixture, Fuel 140 (2015), 201-208.

30. H. Wang, E. Dames, B. Sirjean, D. A. Sheen et al. A high-temperature chemical kinetic model of nalkane (up to $n$-dodecane), cyclohexane, and methyl-, ethyl-, n-propyl and n-butyl-cyclohexane oxidation at high temperatures, JetSurF version 2.0, September 19, 2010 (http://web.stanford.edu/group/haiwanglab/JetSurF/JetSurF2.0/index.html).

31. A.E. Lutz, R.J. Kee, J.A. Miller. SENKIN: A FORTRAN program for predicting homogeneous gas phase chemical kinetics with sensitivity analysis. No. SAND-87-8248. Sandia National Labs. Livermore, CA (USA), 1988. 
32. H. Wang, X.Q. You, A.V. Joshi, et al., 2007, USC Mech Version II. High-Temperature Combustion Reaction Model of $\mathrm{H}_{2} / \mathrm{CO} / \mathrm{C}_{1}-\mathrm{C}_{4}$ Compounds, available at: http://ignis.usc.edu/USC_Mech_II.htm.

33. T. Kohonen, The self-organizing map, Proc. IEEE 78 (1990) 1464-1480.

34. M.A. Springer, S.B. Pope, Exploiting ISAT to solve the reaction-diffusion equation, Combust. Theory Model. 8 (2004) 361-383. 